\title{
2349 Periodontal Risk Spider-web for Periodontal Assessment in Hong Kong Chinese
}

A.C.C. CHAN, L. JIN, and E.F. CORBET, The University of Hong Kong, Hong Kong

Objectives: This preliminary study aimed to build up a practical periodontal risk assessment system in Hong Kong Chinese adults. Methods: A total of 99 Chinese dental hospital attendees (39 males and 60 females) aged 18 to 75 years (mean age: 45.4 14.0 years) participated in this study. They were interviewed using structured questionnaires. Full-mouth periodontal examination was then performed. A personal Periodontal Risk Spider-web (PRS) was formulated including 6 variables: i) cigarette smoking; ii) Diabetes Mellitus; iii) Body Mass Index (BMI); iv) halitosis; v) oral hygiene habits; and vi) dental visit patterns. Three periodontal risk categories were defined following PRS total score calculation: low risk (0-3), moderate risk (4-5) and high risk (>5). The association of each variable ( $\mathrm{i}$ to $\mathrm{vi}$ ) and the PRS total score with the severity of periodontal destruction was determined and relative risk (RR) and odds ratio (OR) were calculated. Results: Mean \% of sites with probing pocket depth (PPD) $\geq 6 \mathrm{~mm}$ in the subjects with smoking habit, Diabetes Mellitus or self-reported halitosis was significantly greater than in those who were free of these conditions $(p<0.05)$. Smokers with halitosis showed 3 -fold greater \% of sites with $P P D \geq 6 \mathrm{~mm}$ than the non-smokers without halitosis $(p<0.05)$. Significantly greater $\%$ of

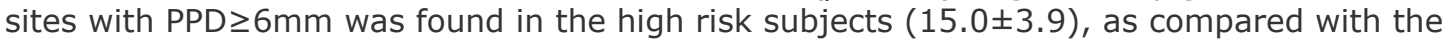
moderate risk subjects $(6.9 \pm 1.4, p<0.02)$ and the low risk subjects $(4.3 \pm 1.1, p<0.01)$. The subjects in the high risk group were significantly more likely $(R R=1.36$; $O R=2.74)$ to exhibit advanced periodontitis than those in the low risk group. Conclusion: This study suggests that the proposed personal Periodontal Risk Spider-web may be promising for periodontal risk assessment in clinical practice for Hong Kong Chinese adults. Supported by Hong Kong Research Grants Council (RGC HKU 7518/05M) \& The University of Hong Kong (CRCG 2005-06).

Seq \#189 - Periodontal Diagnosis 3

3:30 PM-4:30 PM, Friday, 30 June 2006 Brisbane Convention \& Exhibition Centre Exhibit Hall 1

Back to the Periodontal Research - Diagnosis / Epidemiology Program Back to the IADR General Session \& Exhibition (June 28 - July 1, 2006) 